\title{
CREATING WALLONIA'S NEW VERY HIGH RESOLUTION LAND COVER MAPS: COMBINING GRASS GIS OBIA AND OTB PIXEL-BASED RESULTS
}

\author{
M. Lennert ${ }^{* 1}$, T. Grippa ${ }^{1}$, J. Radoux ${ }^{2}$, C. Bassine ${ }^{2}$, B. Beaumont ${ }^{3}$, P. Defourny ${ }^{2}$, E. Wolff ${ }^{1}$ \\ ${ }^{1}$ ANAGEO-DGES, Université Libre de Bruxelles, Belgium, moritz.lennert@ulb.ac.be \\ ${ }^{2}$ Earth and Life Institute, Université catholique de Louvain, Louvain-la-Neuve, Belgium \\ ${ }^{3}$ Remote Sensing and Geodata Unit, Institut Scientifique de Service Public, Liège, Belgium
}

Commission IV, WG IV/4

KEY WORDS: Remote Sensing, Land Cover, Ensemble fusion, Wallonia, GRASS GIS, Orfeo ToolBox

\begin{abstract}
:
The Walloon region of Belgium has launched a research project that aims at elaborating a methodology for automated, high-quality land cover mapping, based primarily on its yearly $0.25 \mathrm{~m}$ orthophoto coverage. Whereas in urban areas an object-based (OBIA) approach has been the privileged path in the last years as it allows taking into account shape information relevant for the characterization of man-made constructions, such an approach has its limits in the rural and more natural areas due to increased difficulties for segmentation and less sharp boundaries, thus calling for a pixel-based approach. The project thus consists in developing a combination of methods, and to integrate their results through an ensemble fusion approach. As many of the more natural land cover classes have temporal profiles which cannot be detected in a one-date orthoimage, Sentinel 1 and 2 data are also included in order to take advantage of their higher spectral and temporal resolution. All methods are trained using existing regional databases. In a second step, we combine the different LC classification results by fusioning them into one high-accuracy (over $90 \%$ $\mathrm{OA}$ ) product, using a series of different approaches ranging from rule-based to machine learning to the Dempster-Shafer method. The entire toolchain is based on free and open source software, mainly GRASS GIS and Orfeo ToolBox. Results indicate the importance of the quality of the individual classifications for the fusion results and justify the choice of combining OBIA and pixelbased approaches in order to avoid the pitfalls of each.
\end{abstract}

\section{INTRODUCTION}

Land cover (LC) maps, showing the characteristics of surface elements, e.g. vegetation, artificial constructions, water, etc, are essential components for regional decision-making, for uses as diverse as spatial planning, environmental monitoring and modelling, flood risk assessment, etc.

Even though the Walloon region in Belgium has compiled a rich catalogue of vector geodata, the actual LC map currently available dates back over a decade and an update was thus needed. The regional administration decided to launch a research project to develop a robust, automatized, scalable and reproducible method for creating these data, mainly based on the available VIS-NIR orthoimagery at $0.25 \mathrm{~m}$ resolution, as well as height information derived through photogrammetry from the raw version of that same imagery. The ultimate aim of the project is not only to provide recent (2018), INSPIREcompliant maps, but also to elaborate a method that would make it easier for the region to reproduce such data at higher temporal frequency than in the past, ideally based on FOSS4G software in order to avoid vendor lock-in and licence costs. As a response to the need for high accuracy in very diversified landscapes, from densely urbanized areas to large forests, a combination of approaches was chosen.

This paper details the preliminary outputs of the work, which is still in progress. We begin with a very short overview of the current state-of-the-art in LC mapping, to then go on to describing the data, methods and intermediate results, before discussing the lessons already learned.

\section{STATE OF THE ART}

\subsection{Different approaches to LC mapping}

Because of its importance for many different fields, land cover mapping has attracted a lot of attention from the research community. Starting with simple pixel-based approaches based on low resolution satellite images available at the time, the advent of very high resolution (VHR) imagery has led to the development of object-based approaches, notably for man-made landscapes such as urban areas (Blaschke 2010; Chen et al. 2018). However, with more and more open data available at global scale, particularly the Landsat and Sentinel satellites, pixel-based methods continue to attract attention and development (Grekousis, Mountrakis, and Kavouras 2015). Such approaches as also particularly used in the environmental sciences community, for example in the work concerning local climate zones (Stewart and Oke 2012; Bechtel et al. 2015), the ESA climate change initiative (Bontemps et al. 2012; Hollmann et al. 2013) or crop mapping (Inglada et al. 2015).

Another, new actor is deep learning which has shown very promising results for land cover mapping (Zhu et al. 2017; Zhang, Zhang, and Du 2016). Deep learning is, however, seen by many as a "black box", making it difficult to use in public decision-making which requires accountability (Dosilovic, Brcic, and Hlupic 2018; Samek, Wiegand, and Müller 2017). This black box character, leading to more difficulties for the administration to assess and accept the proposed processes than classic, well-known methods, was one of the main reasons not to use deep learning in this project.

\footnotetext{
* Corresponding author, moritz.lennert@ulb.ac.be
} 


\section{DEFINITION OF USER NEEDS AND OBJECTIVES}

An important part of the project, although not treated in detail here is the work with users in order to identify and prioritize their needs (Beaumont et al. 2019). This includes the definition of a legend for the final land cover product. As Figure 1 shows, a legend with 6 main classes has been designed, with two of the main classes subdivided into two subclasses each. One of the classes, arable land, was difficult to define because it combines two land cover types in time (bare soil and herbaceous vegetation within the same year). This can obviously not be detected with single-date imagery, calling for the use of images of higher temporal resolution, but lower spatial resolution such as Sentinel 1 and 2.

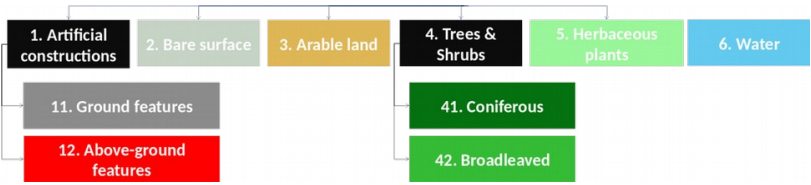

Figure 1: Landcover legend defined after user consultation

Beyond the legend, other issues need to be balanced when working on such land cover maps: the necessary level of accuracy, the temporal resolution and the minimum size of objects in the map (minimum mapping unit or MMU). In order to identify acceptable trade-offs, users were asked to allocate a limited budget of points to these three objectives. Figure 2 shows the result of this consultation, including the contradictory needs. As a general conclusion, it was decided to aim for an MMU of $15 \mathrm{~m}^{2}$ (possibly to be adapted according to classes), a frequency of update between 3 and 5 years, and a minimum overall accuracy of the product over $85 \%$. As this first round of mapping should provide a very solid baseline for future updates, the team has proposed to aim for a much higher overall accuracy (around 95\%).

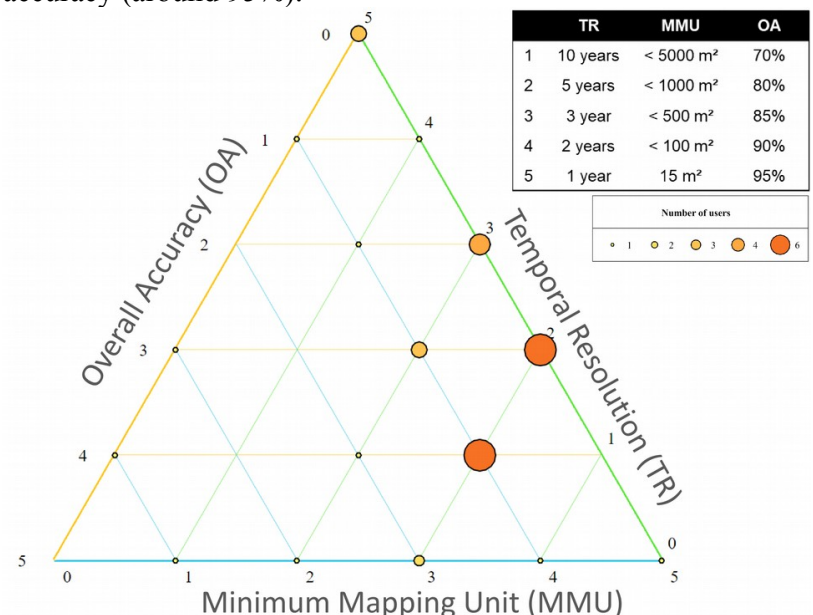

Figure 2: Compromises between objectives as preferred by users

\section{DATA}

Different sources of data are available on the study area. Each has its own spatial reference and specific feature for the land cover classification. The main input is a mosaic of orthophoto images with $25 \mathrm{~cm}$ pixels and 4 spectral bands (NIR-Red-Green and Blue). The study area was covered thanks to several flights in spring and summer 2018. The data provider also used photogrammetry to build a digital surface model from the original photographs. A digital height model was then derived by subtracting a $1 \mathrm{~m}$ resolution LIDAR-based digital elevation model from 2013. Beside the very high resolution airborne datasets, images from Sentinel-1 (10 m C-Band SAR data) and Sentinel-2 (10 and $20 \mathrm{~m}$ multispectral data) are used to analyze the temporal dynamic of the land cover. Finally, a $2 \mathrm{~m}$ open data land cover layer from the Lifewatch project (Radoux et al. 2019) is used for training, as well as a vector database of roads, railways and rivers provided by the Walloon Region. This vector database was enhanced into a planimetric reference : a linear roads dataset and three planimetric datasets for railways, buildings and rivers. From the linear ancillary data, the road network was completed and made continuous using toolboxes from the open source platform QGIS and GRASS GIS. In particular, the linear referencing toolbox from QGIS was used to solve completeness issues in a road dataset.

\section{METHODS}

The methods used were chosen based on an ensemble of criteria:

- $\quad$ Reflecting the state-of-the-art

- Ease of application for a regional administration

- Scalability for very large datasets (the input data is several TB)

- Potential for automation of the entire procedure

- Existing experience in the research teams

\subsection{Per pixel approach using Orfeo ToolBox}

Orfeo ToolBox (OTB) is a C++ library for state of the art remote sensing (Grizonnet et al. 2017). All of the algorithms are accessible from Monteverdi, QGIS, Python, the command line or C++. OTB is also the core of the SEN2AGRI toolbox (http:// www.esa-sen2agri.org/), an image processing platform used in this study for the classification of crop types based on Sentinel2 images. Due to the large size of the orthophotos, command lines are managed by the SLURM job scheduling system. The parallel processing is driven by zones of similar flight conditions according to the metadata of the orthophotos.

The first step of the processing consists in smoothing the orthophotos based on the meanshift algorithm (Comaniciu and Meer 2002) in order to reduce potential salt and pepper effects. A reference dataset is then built based on the $2 \mathrm{~m}$ land cover map, the MNH and the shadows predicted from the MNS with a custom OTB application. This reference dataset is then eroded using a multiclass mathematical morphology operator (Radoux et al. 2014). The orthophotos are then classified with an a priori probability defined by the height class. In addition, a random forest classifier was applied on a stack of two dates (leaves on and leaves off) of Sentinel-2 images. This classification focuses on the discrimination of forest types to better discriminate between broadleaved deciduous and needle-leaved evergreen forests, as well as larch stands which are deciduous needleleaved trees.

\subsection{OBIA approach using GRASS GIS}

GRASS GIS is a full-fledged geographical information system (Neteler et al. 2012), grown over 35 years of continuous development. The OBIA approach used here is based on a complete toolchain developed during the last years which provides everything from segmentation (including unsupervised parameter optimization) to machine learning classifiers (Grippa et al. 2017). All tools have been specifically designed for very large data sets, allowing parallel computing. A previous project already allowed a first test of this toolchain on a small part of the Walloon territory (Beaumont et al. 2017). 
Recent studies have shown that using a constant segmentation parameter across space does not lead to ideal segments, as spatial structures differ significantly between different parts of the territory (Grippa et al. 2017; Georganos et al. 2018; Drăguț et al. 2019). The OBIA approach in this study takes this into account by optimizing the segmentation within fairly small tiles, delineated using a module for the creation of semantically useful cutlines (Lennert and GRASS GIS Development Team 2018), inspired by the work of Soares et al (2016). In order to speed up the region-growing segmentation, superpixels are first created in a rapid run of the SLIC algorithm (Kanavath, Metz, and GRASS GIS Development Team 2018; Achanta et al. 2012).

After segmentation, diverse statistics (spectral, shape, texture, height, $\mathrm{x}$ and $\mathrm{y}$ coordinates) are gathered for each segment, including information about the segments neighbours. Using the existing vector databases, segments falling into polygons of specific classes are identified automatically as training segments. Outliers (e.g. segments identified as artificial construction which have a tree growing over them in the image) are eliminated through simple tests mainly based on NDVI and height.

The data set is divided into strata according to the date at which the photos were taken. A subsample of the very large set of training segments is then used to train a different random forest model on each of the strata and the resulting models applied on each strata's tiles, resulting in a choice of class for each segment, as well as the probability of each class. The OBIA approach focuses specifically on the quality of the classification of more man-made landscapes, for which it is particularly well suited.

\subsection{Data fusion}

We test three different approaches for the fusion of the raw LC maps into one final automated product. A set of 1500 points labelled by visual interpretation and stratified in 3 strata to cover different types of mismatches between the input classifications, is used as a reference for training and validation. At the time of writing, this fusion is still in progress, and only preliminary results are presented here. Beyond ongoing work on the different input classifications, we are currently elaborating an enhanced point dataset in order to improve the model training.

\subsubsection{Dempster-Shafer}

The different classification results and the clean vector database are fused at the pixel level based on the Dempster-Shafer fusion (Ran et al. 2012). This method combines masses of belief based on the confusion matrix of each classification. The mass of belief indicates the belief of each input classification present for each label value based on a reference dataset. The confusion matrix is computed independently for three strata selected based on the likelihood of mislabelling. For each strata in the reference point data set, a specific fusion is computed and the three results are merged by an ultimate Dempster-Shafer fusion based on the entire set of points.

\subsubsection{Object-based machine learning}

The fusion in this approach is based on the objects created through the OBIA classification. For each of these objects a series of features are extracted from the different classification attempts such as entropy-based analysis of class probabilities from both the per-pixel and the OBIA results, class proportions within each object from the pixel-based results, modal classes of the Sentinel-based results as well as modal classes from binary version of the ancillary vector datasets.

Using the reference points a random forest classifier is then trained to predict classes for the objects.

\subsubsection{Rule-based}

The rule-based approach is built interactively based on expert assessment via visual interpretation. It currently comprises about 15 conditional, pixel-based rules. For each pixel $(25 \times 25 \mathrm{~cm}), \mathrm{LC}$ is attributed taking into account the agreement between the two ortho-image classifications, the presenceabsence within ancillary reference datasets and contextual rules. The Sentinel-based classifications (time-series) and digital height model serve as support for the LC allocation of complex remaining pixels.

Knowing that such as rule-based approach requires significant investment of human resources to arrive at satisfying results over a very large territory, we mainly consider it as a reference application which allows us to benchmark the other approaches.

\section{RESULTS}

\subsection{Results of different classifications}

In this section we present a small selection of results of the VHR pixel-based and OBIA approaches in order to illustrate some difficulties in each. Figure 3 shows some typical issues of pixel-based approaches: on the left, one can clearly see the saltand-pepper effect linked to the high variability of individual spectral signatures, while the extract on the right illustrates the high sensitivity to the quality (and spatial precision) of the input data, in this case the height information which does not have the same precision as the spectral data, and is slightly spatially shifted.

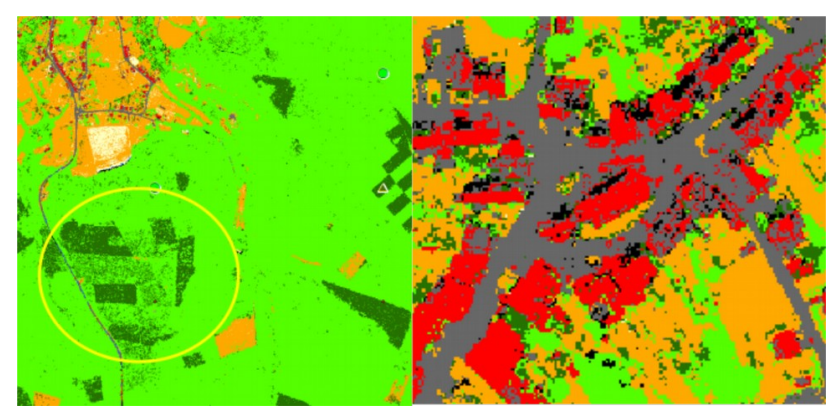

Figure 3: Illustration of issues in the pixel-based classification

While objects resulting from an OBIA approach often allow mitigating the issue of high variability of individual pixelvalues by using means or other aggregated statistics, figure 4 illustrates quite well a fundamental issue with object-based approaches in very heterogeneous environments such as forests. Objects in these regions are often too small, increasing the risk of misclassification.

Figure 5 reinforces this idea by showing that objects which are not as easily segmented as buildings have a higher uncertainty in their classification results. At the same time, the figure demonstrates the usefulness of the OBIA technique for delineating man-made structures fairly precisely. 


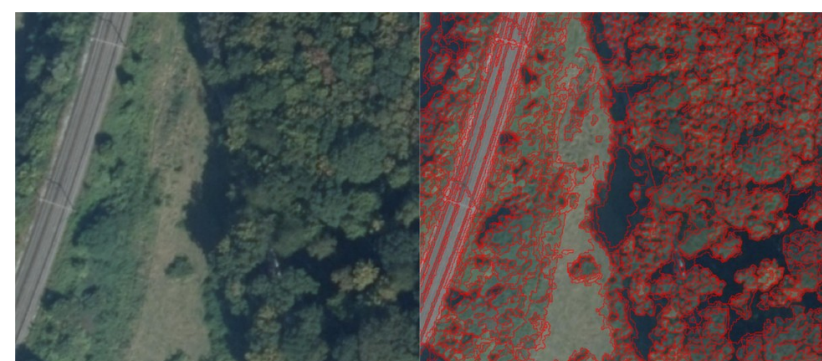

Figure 4: Illustration of over-segmentation in the OBIA approach

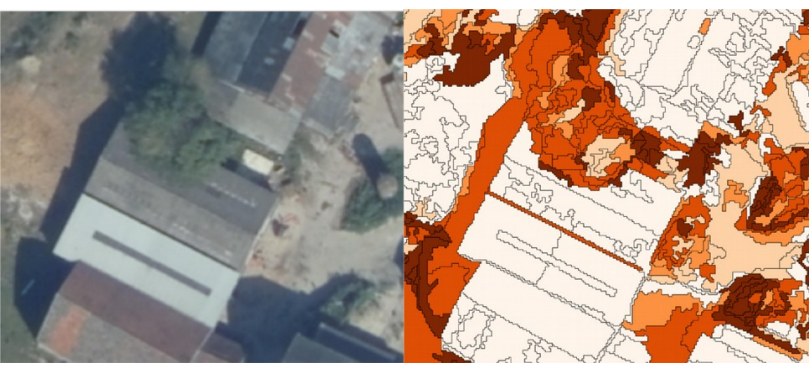

Figure 5: Illustration of uncertainty linked to object type in OBIA (darker color $=$ higher uncertainty)

\subsection{Results of fusion}

As mentioned above, the work on fusion is still ongoing, but some first results already are available and allow for an initial analysis of the advantages or disadvantages of the different approaches, as illustrated in figures 6-9 (see figure 1 for the legend).

A major difference can be seen between the object-based, machine learning approach and the two others is the fact that just as in the initial classification, the objects provide a certain smoothing effect, while ensuring sharper edges for man-made constructions. The Dempster-Shafer approach has difficulties dealing with the different resolutions and thus leads to a less spatially precise result, although thematically it sometimes does a better job identifying the right classes.

One class which is difficult to deal with in the entire processing chain is arable land. As the VHR classifications are limited to one point in time, this information can only come from the Sentinel-derived classifications which have much lower resolution.

\subsection{Discussion}

The above results show that fusion clearly provides qualitative improvements over the individual classifications. However, a major determinant of the quality of results is the accuracy of the inputs into the fusion. This is why the team adopted an iterative approach, going back and forth between fusion and original classification in order to identify the best parameters at each step.

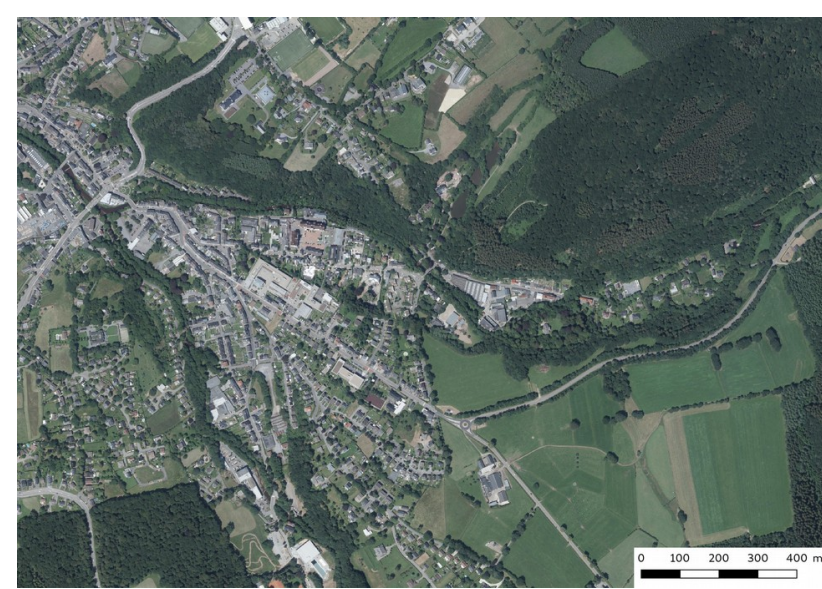

Figure 6: Original orthophoto

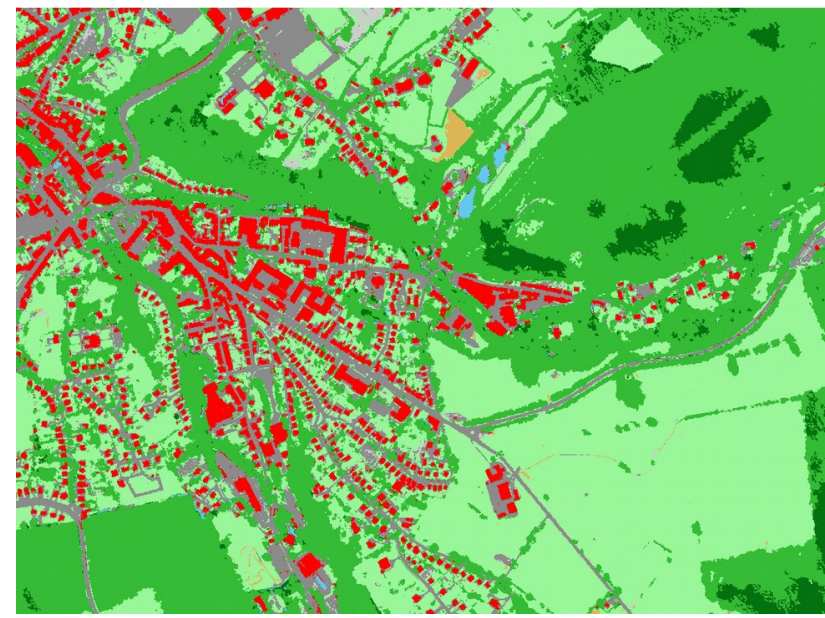

Figure 7: Object-based fusion using machine learning (for legend see figure 1)

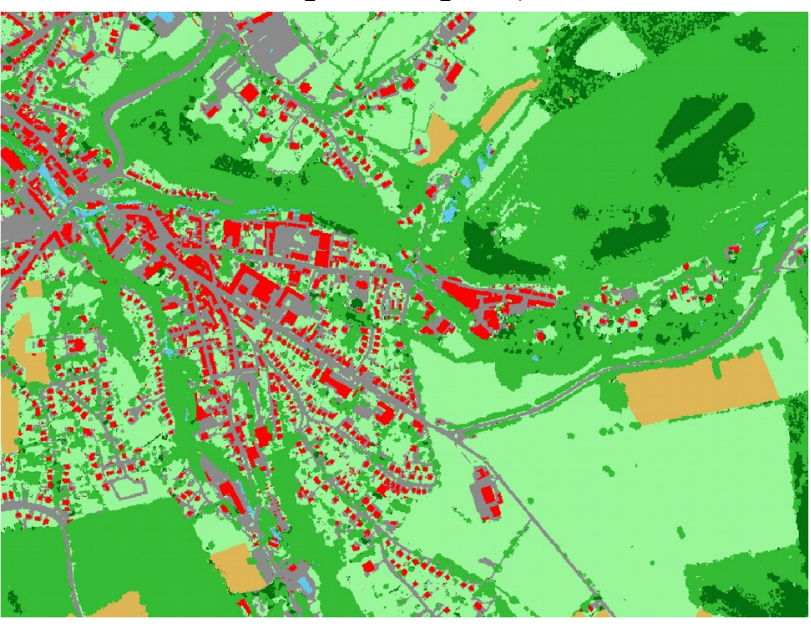

Figure 8: Rule-based fusion ((for legend see figure 1) 


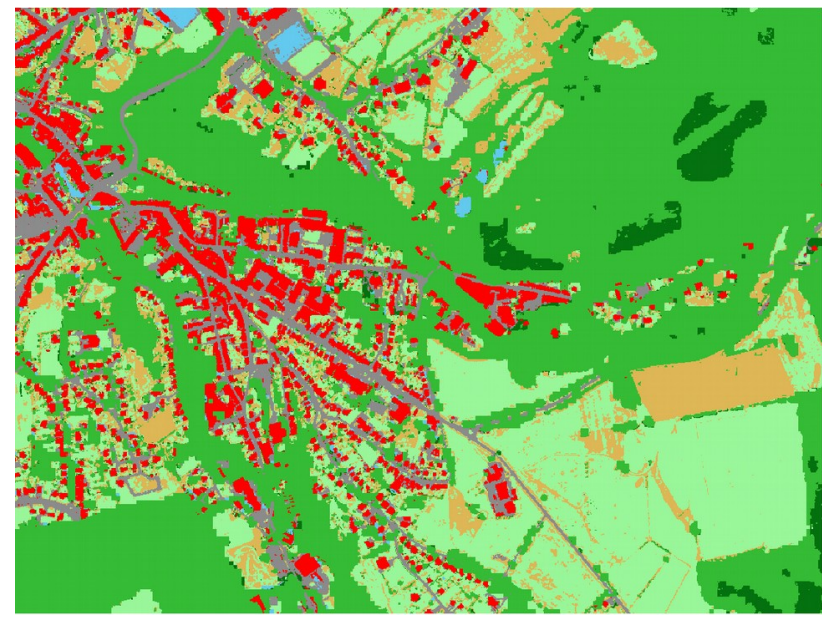

Figure 9: Dempster-Shafer fusion (for legend see figure 1)

Giving priority to the ancillary reference datasets provides smoother maps for these features, but strongly depends on the timeliness of their content (e.g. lots of omission/commission errors if not up to date, such as for the building layer). This is somewhat of a chicken-and-egg problem, as a procedure for elaborating a high quality LC map depends on up-to-date input, while potentially being one of the prime sources of such up-todate information.

As expected, the rule-based approach is time-consuming, notably for the parametrization of the rules, as well as subjective, so it is difficult to implement in an automated setting across a large territory.

\section{CONCLUSION AND PERSPECTIVES}

While the precise details of classification and fusion are still work in progress, we can already clearly see that using multiple data, classifying them through different methods and combining them in a final fusion step leads to better results than the individual methods on their own. The results seem on par with the precision level expected by the potential users, and the entire processing chain can potentially be in-housed within the administration as it relies entirely on free and open source software and simple scripts that ensure complete automation. The final LC map will be made available as open data through the Walloon region's geoportal.

An important aspect of the project for the FOSS4G community is the fact that it has allowed continuous improvement of existing tools. Some GRASS GIS modules were enhanced during the project (e.g. i.segment.uspo, i.segment.stats, i.cutlines, v.class.mlR), with all enhancements integrated back into the software's source code. Some new modules were also developed (e.g. r.texture.tiled) and are now available for the entire community. This clearly shows the advantage for public administrations to fund projects based on FOSS4G as all developments will continue to be available, including to other parts of the administration, thus providing a potential effect of pooling of public resources.

Within the project itself work will continue in order to finalize the automated LC map. A period of manual corrections is foreseen in order to create one extremely high quality base map which can provide the foundation of a high frequency update cycle of LC maps in Wallonia. Furthermore, the project will integrate LC information as attribute data into existing regional vector databases, thus supporting easier uptake of the data by different user communities, and a combination of the LC map with existing alpha-numeric, geocoded, databases will provide input into automated land use (LU) mapping.

Future research will have to confront the methods developed here to new developments, notably in deep learning. As mentioned in the literature review, some elements of deep learning make it less attractive to public administrations. However, the very high quality LC map coming out of this project has the potential to provide useful input for the training of deep learning networks, possibly maintained within the administration for future needs. Although this will require an effort to make the entire process less black box, it has the potential of providing the administration with a potent tool for frequent updating.

\section{ACKNOWLEDGEMENTS}

The authors would like to acknowledge the Walloon Government for the funding of the project WALOUS.

Computational resources for the OBIA and machine learning fusion approaches are being provided by the Shared ICT Services Centre, Université Libre de Bruxelles.

Computational resources for the pixel-based processing have been provided by the Consortium des Équipements de Calcul Intensif (CÉCI), funded by the Fonds de la Recherche Scientifique de Belgique (F.R.S.-FNRS) under Grant No. 2.5020 .11 and by the Walloon Region.

The authors would also like to thank the GRASS GIS Development team for their responsiveness to any requests for changes.

\section{REFERENCES}

Achanta, R., A. Shaji, K. Smith, A. Lucchi, P. Fua, and Sabine Süsstrunk. 2012. "SLIC Superpixels Compared to State-of-theArt Superpixel Methods." IEEE Transactions on Pattern Analysis and Machine Intelligence 34 (11): 2274-82. https://doi.org/10.1109/TPAMI.2012.120.

Beaumont, Benjamin, Tais Grippa, Moritz Lennert, Sabine Vanhuysse, Nathalie Stephenne, and Eléonore Wolff. 2017. "Toward an Operational Framework for Fine-Scale Urban Land-Cover Mapping in Wallonia Using Submeter Remote Sensing and Ancillary Vector Data." Journal of Applied $\begin{array}{lcc}\text { Remote } & \text { Sensing } & 11 \\ \text { https://doi.org/10.1117/1.JRS.11.036011. }\end{array}$

Beaumont, Benjamin, Laura Van de Vyvere, Nathalie Stephenne, Coraline Wyard, and Eric Hallot. 2019. 'Users' Consultation Process in Building a Land Cover and Land Use Database for the Official Walloon Georeferential." presented at the Joint Urban Remote Sensing Event (JURSE), Vannes.

Bechtel, Benjamin, Paul J. Alexander, Jürgen Böhner, Jason Ching, Olaf Conrad, Johannes Feddema, Gerald Mills, Linda See, and Iain Stewart. 2015. "Mapping Local Climate Zones for a Worldwide Database of the Form and Function of Cities." ISPRS International Journal of Geo-Information 4 (1): 199219. https://doi.org/10.3390/ijgi4010199.

Blaschke, T. 2010. "Object Based Image Analysis for Remote Sensing." ISPRS Journal of Photogrammetry and Remote Sensing $\quad 65 \quad$ (1): 2-16. https://doi.org/10.1016/j.isprsjprs.2009.06.004. 
Bontemps, S., M. Herold, L. Kooistra, A. van Groenestijn, A. Hartley, O. Arino, I. Moreau, and P. Defourny. 2012. "Revisiting Land Cover Observation to Address the Needs of the Climate Modeling Community." Biogeosciences 9 (6): 2145-57. https://doi.org/10.5194/bg-9-2145-2012.

Chen, Gang, Qihao Weng, Geoffrey J. Hay, and Yinan He. 2018. "Geographic Object-Based Image Analysis (GEOBIA): Emerging Trends and Future Opportunities." GIScience \& Remote Sensing $\quad 55 \quad$ (2): $\quad 159-82$. https://doi.org/10.1080/15481603.2018.1426092.

Comaniciu, D., and P. Meer. 2002. "Mean Shift: A Robust Approach toward Feature Space Analysis." IEEE Transactions on Pattern Analysis and Machine Intelligence 24 (5): 603-19. https://doi.org/10.1109/34.1000236.

Dosilovic, Filip Karlo, Mario Brcic, and Nikica Hlupic. 2018. "Explainable Artificial Intelligence: A Survey." In 201841 st International Convention on Information and Communication Technology, Electronics and Microelectronics (MIPRO), 0210 $15 . \quad$ Opatija: IEEE. https://doi.org/10.23919/MIPRO.2018.8400040.

Drăguț, Lucian, Mariana Belgiu, George Popescu, and Peter Bandura. 2019. "Sensitivity of Multiresolution Segmentation to Spatial Extent." International Journal of Applied Earth Observation and Geoinformation 81 (September): 146-53. https://doi.org/10.1016/j.jag.2019.05.002.

Georganos, Stefanos, Tais Grippa, Moritz Lennert, Sabine Vanhuysse, Brian Johnson, and Eléonore Wolff. 2018. "Scale Matters: Spatially Partitioned Unsupervised Segmentation Parameter Optimization for Large and Heterogeneous Satellite Images." Remote Sensing $10 \quad$ (9): 1440. https://doi.org/10.3390/rs10091440.

Grekousis, George, Giorgos Mountrakis, and Marinos Kavouras. 2015. "An Overview of 21 Global and 43 Regional Land-Cover Mapping Products." International Journal of $\begin{array}{llll}\text { Remote } & \text { Sensing } & 36 & \text { (21): }\end{array}$ https://doi.org/10.1080/01431161.2015.1093195.

Grippa, Tais, Stefanos Georganos, Moritz Lennert, Sabine Vanhuysse, and Eléonore Wolff. 2017. “A Local Segmentation Parameter Optimization Approach for Mapping Heterogeneous Urban Environments Using VHR Imagery.” In . Vol. 10431. Warsaw: International Society for Optics and Photonics. https:// doi.org/10.1117/12.2278422.

Grippa, Taïs, Moritz Lennert, Benjamin Beaumont, Sabine Vanhuysse, Nathalie Stephenne, and Eléonore Wolff. 2017. "An Open-Source Semi-Automated Processing Chain for Urban Object-Based Classification." Remote Sensing 9 (4): 358. https://doi.org/10.3390/rs9040358.

Grizonnet, Manuel, Julien Michel, Victor Poughon, Jordi Inglada, Mickaël Savinaud, and Rémi Cresson. 2017. "Orfeo ToolBox: Open Source Processing of Remote Sensing Images." Open Geospatial Data, Software and Standards 2 (1): 15. https://doi.org/10.1186/s40965-017-0031-6.

Hollmann, R., C. J. Merchant, R. Saunders, C. Downy, M. Buchwitz, A. Cazenave, E. Chuvieco, et al. 2013. "The ESA Climate Change Initiative: Satellite Data Records for Essential Climate Variables." Bulletin of the American Meteorological Society 94 (10): 1541-52. https://doi.org/10.1175/BAMS-D-1100254.1.

Inglada, Jordi, Marcela Arias, Benjamin Tardy, Olivier Hagolle, Silvia Valero, David Morin, Gérard Dedieu, et al. 2015. "Assessment of an Operational System for Crop Type Map
Production Using High Temporal and Spatial Resolution Satellite Optical Imagery." Remote Sensing 7 (9): 12356-79. https://doi.org/10.3390/rs70912356.

Kanavath, R., M. Metz, and GRASS GIS Development Team. 2018. I.Superpixels.Slic. Geographic Resources Analysis Support System (GRASS) Software. USA: Open Source Geospatial Foundation. https://grass.osgeo.org/grass72/manuals/addons/i.superpixels.sli c.html.

Lennert, Moritz, and GRASS GIS Development Team. 2018. I.Cutlines. Geographic Resources Analysis Support System (GRASS) Software. USA: Open Source Geospatial Foundation. https://grass.osgeo.org/grass72/manuals/addons/i.cutlines.html.

Neteler, Markus, M. Hamish Bowman, Martin Landa, and Markus Metz. 2012. "GRASS GIS: A Multi-Purpose Open Source GIS.” Environmental Modelling \& Software 31 (May): 124-30. https://doi.org/10.1016/j.envsoft.2011.11.014.

Radoux, Julien, Axel Bourdouxhe, William Coos, Marc Dufrêne, and Pierre Defourny. 2019. "Improving Ecotope Segmentation by Combining Topographic and Spectral Data." $\begin{array}{lllll}\text { Remote } & \text { Sensing } & 11 & \text { (3): } & 354 .\end{array}$ https://doi.org/10.3390/rs11030354.

Radoux, Julien, Céline Lamarche, Eric Van Bogaert, Sophie Bontemps, Carsten Brockmann, and Pierre Defourny. 2014. "Automated Training Sample Extraction for Global Land Cover Mapping." Remote Sensing 6 (5): 3965-87. https://doi.org/10.3390/rs6053965.

Ran, Y.H., X. Li, L. Lu, and Z.Y. Li. 2012. "Large-Scale Land Cover Mapping with the Integration of Multi-Source Information Based on the Dempster-Shafer Theory." International Journal of Geographical Information Science 26 (1): 169-91. https://doi.org/10.1080/13658816.2011.577745.

Samek, Wojciech, Thomas Wiegand, and Klaus-Robert Müller. 2017. "Explainable Artificial Intelligence: Understanding, Visualizing and Interpreting Deep Learning Models." ArXiv:1708.08296 [Cs, Stat], August. http://arxiv.org/abs/1708.08296.

Soares, Anderson Reis, Thales Sehn Körting, and Leila Maria Garcia Fonseca. 2016. "IMPROVEMENTS OF THE DIVIDE AND SEGMENT METHOD FOR PARALLEL IMAGE SEGMENTATION." Revista Brasileira de Cartografia 68 (6). http://www.lsie.unb.br/rbc/index.php?

journal $=$ rbc\&page $=$ article \&op $=$ view $\&$ path $\% 5 B \% 5 \mathrm{D}=1602$.

Stewart, I. D., and T. R. Oke. 2012. "Local Climate Zones for Urban Temperature Studies." Bulletin of the American Meteorological Society $93 \quad$ (12): 1879-1900. https://doi.org/10.1175/BAMS-D-11-00019.1.

Zhang, L., L. Zhang, and B. Du. 2016. "Deep Learning for Remote Sensing Data: A Technical Tutorial on the State of the Art." IEEE Geoscience and Remote Sensing Magazine 4 (2): 22-40. https://doi.org/10.1109/MGRS.2016.2540798.

Zhu, Xiao Xiang, Devis Tuia, Lichao Mou, Gui-Song Xia, Liangpei Zhang, Feng Xu, and Friedrich Fraundorfer. 2017. "Deep Learning in Remote Sensing: A Comprehensive Review and List of Resources." IEEE Geoscience and Remote Sensing Magazine $\quad 5 \quad$ (4): 8-36. https://doi.org/10.1109/MGRS.2017.2762307. 
The International Archives of the Photogrammetry, Remote Sensing and Spatial Information Sciences, Volume XLII-4/W14, 2019 FOSS4G 2019 - Academic Track, 26-30 August 2019, Bucharest, Romania

\section{APPENDIX}

All scripts used for the OBIA part of the project are made available on https://github.com/mlennert/WALOUS.

Revised June 2019 